\title{
Hg 5d and 6s: Multichannel quantum-defect analysis of experimental data
}

\author{
F. Schäfers \\ Berliner Elektronenspeicherring-Gesellschaft für Synchrotonstrahlung mbH, Lentzeallee 100, D-1000 Berlin 33, West Germany \\ Ch. Heckenkamp \\ Gesellschaft für Automation und Organisation, D-8000 München 70, West Germany \\ M. Müller \\ Fritz-Haber-Institut der Max-Planck-Gesellschaft, D-1000 Berlin 33, West Germany \\ and Fakultät für Physik, Universität Bielefeld, D-4800 Bielefeld, West Germany \\ V. Radojević \\ University of Virginia, Charlottesville, Virginia \\ U. Heinzmann \\ Fritz-Haber-Institut der Max-Planck-Gesellschaft, D-1000 Berlin 33, West Germany \\ and Fakultät für Physik, Universität Bielefeld, D-4800 Bielefeld, West Germany \\ (Received 6 September 1989; revised manuscript received 22 February 1990)
}

\begin{abstract}
Experimental spin-polarization data for the $\mathrm{Hg} 6 s$ Cooper minimum and dipole-transition amplitudes and phase-shift differences for photoionization of the $\mathrm{Hg} 5 d$ and $6 s$ shells (eight channels) in the photon-energy region from the ${ }^{2} D_{3 / 2}$ threshold up to $35 \mathrm{eV}$ (kinetic energies from 0 to $20 \mathrm{eV}$ ) are presented and compared with new relativistic random-phase approximation calculations. The data were evaluated from an experimental data set, quantum mechanically "complete," consisting of cross-section and photoelectron spin-polarization data. For the $5 d$ subshells the results show strong interchannel coupling between the outgoing $p$ and $f$ continuum channels. The results for $\mathrm{Hg} 6 s$ could be used for an independent determination of the photoelectron angular distribution parameter $\beta$ in the Cooper-minimum region. This region is shown to be perturbed by numerous two-electron excitations, which might be a possible explanation for the controversy on its location.
\end{abstract}

\section{INTRODUCTION}

This paper presents experimental and theoretical data on the low-energy photoionization of mercury atoms. As a high- $Z$ closed-shell atom, $\mathrm{Hg}$ (outer shell configuration $5 d^{10} 6 s^{2}$ ) has received much attention in the last years: The relativistic (i.e., spin-orbit) effects, the many-electron effects in terms of inter- and intrashell correlations, and the influence of the potential barrier on the outgoing $l \rightarrow l+1$ waves can be studied on a number of experimental observables. Nevertheless, the understanding of the photoionization dynamics is far from being complete.

In the photoionization of $n s^{2}$ subshells, the spin-orbit interaction in the continuous spectrum leads to different wave functions for photoelectron states corresponding to $\epsilon p_{1 / 2}$ and $\epsilon p_{3 / 2}$ outgoing partial waves. Since in these processes neither the initial state nor the final ionic state shows spin-orbit splitting, the spin-orbit interaction in the continuum appears in its "pure" form. Recently, nssubshell ionization for the heavier noble gases and the group-IIb elements has been investigated in the open continuum both theoretically ${ }^{1-7}$ and experimentally ${ }^{8-13}$ and has been found to be strongly influenced by interchannel interactions. Theoretical calculations have used highly sophisticated methods to include many-body effects in random-phase approximation with exchange (RPAE), 1,4 relativistic random-phase approximation (RRPA), ${ }^{2,3,5}$ and relativistic time-dependent local-density approximation (RTDLDA). ${ }^{6,7}$ A comparison of the theoretical with experimental data showed the enormous influence of correlations upon the energy dependence of the cross section, the photoelectron angular distribution, and the photoelectron spin polarization.

In $\mathrm{Hg} 6 s$ - ionization, special attention has been paid to the position of the Cooper minimum in the vicinity of the $5 d$ threshold. Direct measurement of the cross section ${ }^{9}$ clearly reveals a minimum at about $20-\mathrm{eV}$ photon energy, well above the ${ }^{2} D_{3 / 2}$ threshold. Sophisticated theoretical approaches, ${ }^{1-7}$ however, uniformly yield the minimum in the cross section clearly below the $D$ threshold. To study this problem in more detail, the photoelectron angular distribution, which is described by the $\beta$ parameter and one of the spin-polarization parameters was measured at three rare-gas resonance lines with unpolarized light. ${ }^{10}$ The results supplied important information on the sign of the phase-shift difference in the threshold region, but were not sufficient to give an independent confirmation of 
the measured position of the cross-section minimum. Subsequently, the critical photon-energy region was scanned in $\beta$ measurements with synchrotron radiation, ${ }^{13}$ resulting in a minimum in $\beta$ above the ${ }^{2} D_{3 / 2}$ threshold. This is consistent with the energy dependence of $\beta$ that we had deduced from our first spin-polarization measurements with circularly polarized synchrotron radiation near the $D$-threshold region. ${ }^{14}$

For $\mathrm{Hg} 5 d$ photoionization the spin-orbit interaction leads to two final ionic states with a fine-structure splitting of $1.84 \mathrm{eV}$ between the ${ }^{2} D_{5 / 2}$ and the ${ }^{2} D_{3 / 2}$ ionization thresholds at $14.84-$ and $16.72-\mathrm{eV}$ photon energy, respectively. Since the results of experimental investigations of the cross section ${ }^{9}$ and the $\beta$ parameter $^{13,15}$ as well as all three spin-polarization parameters ${ }^{16,17}$ are now available, a combination of all five dynamical parameters allows a complete characterization of the photoionization process in terms of dipole matrix elements and thus a more sensitive test to theories.

Nonrelativistic calculations of the $\mathrm{Hg} 5 d$ cross section in the Hartree-Slater (HS) and Hartree-Fock (HF) approximations are reported in Refs. 18. The importance of spin-orbit (relativistic) effects in $\mathrm{Hg}$ was pointed out in Refs. 19 and 20 where partial cross sections and angular distributions ${ }^{20}$ using the Dirac-Slater (DS) approximation are reported. In Ref. 21 Dirac-Fock (DF) calculations for $\mathrm{Cd}$ and $\mathrm{Hg}$ are reported. A correlated calculation was performed for outer $6 s$ and $5 d$ subshells in the RRPA formalism in Ref. 5 and for intermediate $5 d, 5 p$, and $4 f$ subshells in Ref. 22. Another correlated calculation of the photoionization for the two outermost subshells in the RTDLDA code was done in Ref. 8. All theories reproduce the general energy dependence fairly well. However, some quantitative mismatch to the experimental data remains, especially in the threshold region that is most sensitive to the coupling schemes and theoretical approaches used. Also, outside the threshold region the interplay between the spin-orbit interaction in the continuum states (which according to Ref. 19 dominates in the $j=3 / 2$ level because it is acting on two weak $p$ waves) and the potential barrier effects (which are more pronounced in the $j=5 / 2$ level because of two outgoing strong $f$ waves) leads to discrepancies.

The response of the experimental observables produced by these effects is rather complex and cannot be simply differentiated. Therefore an analysis of $\mathrm{Hg} 5 d$ experimental data on the basis of the $L S$-coupling scheme was performed. ${ }^{23}$ The results of this analysis revealed strong interchannel interaction between $5 d \rightarrow \epsilon f$ and $5 d \rightarrow \epsilon p$ channels. However, the effects of the spin-orbit interaction in the continuum channels could not be determined because of insufficient experimental data. This relativistic effect manifests itself by introducing a separation of the electron continuum amplitude of angular momentum $l$ into two amplitudes with total angular momentum $j=l \pm 1 / 2$.

The theoretical values with which the experimental data are compared were obtained using the RRPA (Ref. 24) which takes into account a considerable amount of important electron-electron correlations, including coupling between the various photoionization channels. The present calculation is essentially the same as the previous RRPA, ${ }^{5}$ but in the present work we concentrate on the results for dipole-transition amplitudes and excited orbital phase shifts, not reported previously. The calculation was performed in the dipole approximation using a truncated version of the RRPA including and coupling only those channels corresponding to excitation of the $5 d$ and $6 s$ outer shells. There are eight such $j j$-coupled channels ${ }^{5}$ (see Sec. III and Table II for a list of these channels).

It was found that contributions from channels obtained by exciting the $5 p$ shell are very small in the considered energy region., 52 Thus the excitations from the $5 p$ and other inner shells are neglected in a truncated RRPA used before ${ }^{5}$ and in the present work. The omission of these inner channels results in the loss of gauge independence, so that dipole amplitudes have different values in length and in velocity form. Nevertheless, owing to the small contribution of the neglected channels, the two gauges, length and velocity, agree to within a few percent throughout the energy range considered. ${ }^{5}$

The theoretical threshold values in the strict randomphase approximation (RPA) are equal to its Hartree-Fock values, ${ }^{25}$ or in the case of the RRPA equal to the DiracHartree-Fock (DHF) values. ${ }^{24}$ These theoretical values differ from the experimental thresholds because manybody effects are not included in the RPA calculations. As discussed in the literature ${ }^{26}$ certain many-body effects omitted in the strict RPA calculations may be accounted for empirically by using experimental values instead of the HF (or DHF) thresholds. Such a semiempirical procedure has been used in previous calculations ${ }^{5}$ as well as in the present calculation, replacing DHF values by experimental thresholds for $5 d$ and $6 s$ subshells.

In this paper we report in Sec. II our experimental values for the $6 s$ photoionization parameters (crosssection, asymmetry parameter $\beta$, and spin-polarization parameters) in the energy region around and above the $5 d$ thresholds. These data are compared with other experimental measurements and with the results of the RRPA calculations. In the same section (Sec. II) we comment on some conspicious observations of a satellite peak in the photoelectron spectrum, which leads to a discussion of possible influences of two-electron excitations upon the photoionization parameters. Details of our procedure for the determination of multichannel quantum-defect theory (MQDT) parameters (transition matrix elements and phase shifts for each channel) from experimental data for photoionization of $6 s$ and $5 d$ shells are presented in Sec. III. The results of our determination for the transition matrix elements and the relative phase shifts from experimental data are reported in Sec. III A for channels arising from the $6 s$ shell, and in Sec. III B for channels originating from the $5 d$ shell. The experimental data used for the $6 s$ shell are presented in Sec. II, while for the $5 d$ shell the combination of recently published data on the $\beta$ parameter $^{13,15}$ and on the photoelectron spin polarization $^{16,17}$ are used. The evaluated transition matrix elements and the relative phase shifts are compared with the results of the present RRPA calculation. Section IV is devoted to a discussion of the results and concluding remarks. 


\section{EXPERIMENTAL RESULTS FOR Hg $6 s$ COOPER MINIMUM}

The experiments were carried out at the Berlin electron storage ring BESSY using the circularly polarized light of the $6.5-\mathrm{m}$ normal incidence monochromator. ${ }^{27}$ Details of the experimental setup for the measurement of the spinpolarization parameters $A$ (Fano-effect parameter; component in the direction of the light helicity), $\alpha$ (the angular dependence of $A$ ), and $\xi$ (the component perpendicular to the reaction plane) are published elsewhere. ${ }^{28,29}$ In Fig. 1 the experimental results for the spin parameters $A$ and $\xi$ measured with circularly polarized synchrotron radiation are shown (closed circles) together with the earlier data for $\xi$ (Ref. 10) using resonance line radiation (open squares).

A single measurement has been taken in the energy region between the two $D$ thresholds at $15.3-\mathrm{eV}$ photon energy $(\lambda=81 \mathrm{~nm})$. At this particular wavelength, the influence of autoionization resonances upon the cross section is relatively small. ${ }^{30}$ For the spin parameter $A$, several other values exist for the autoinization region directly below the ${ }^{2} D_{5 / 2}$ threshold. The data vary between 0.4 and 0.5 in this narrow regime where individual members of the Rydberg series ${ }^{2} D_{5 / 2} n p_{3 / 2}$ could not be resolved experimentally. ${ }^{30}$

The data in Fig. 1 clearly show negative values and a minimum in $A$ above the ${ }^{2} D_{3 / 2}$ threshold, a sharp increase in $A$ towards lower energies, and a change of sign close to the ${ }^{2} D_{3 / 2}$ threshold.

The spin parameter $\xi$ also varies quite dramatically with photon energy, increasing from values of about 0.1 around $25-\mathrm{eV}$ photon energy up to the maximum of 0.4 approximately $1 \mathrm{eV}$ above the ${ }^{2} D_{3 / 2}$ threshold, followed by a sudden decrease through the region between the two ${ }^{2} D$ thresholds. We note, however, that the maximum in $\xi$ is sharper and appears at lower photon energy than the minimum in $A$.

The data available for the differential cross-section $\sigma$ for $\mathrm{Hg} 6 s$ photoionization, ${ }^{9}$ showing the Cooper minimum at about 20-eV photon energy, and its angular distribution parameter $\beta$ (Refs. 8, 10, and 13) are also depicted in Fig. 1. For $\beta$ and $\alpha$, we give the values calculated from the spin parameters $A$ and $\xi$ (closed circles, see Sec. III A). Direct measurements of $\beta$ using resonance line radiation (open squares, ${ }^{10}$ open triangle ${ }^{8}$ ) and utilizing synchrotron radiation with a bandwidth of $0.16 \mathrm{~nm}$ (open circles ${ }^{13}$ ) are also given. Above 19-eV photon energy, the different values are in good agreement with each other, whereas near the minimum in $\beta$ at about $1 \mathrm{eV}$ above the ${ }^{2} D_{3 / 2}$ threshold the data directly measured by Ref. 13 are systematically lower compared to the other values given, similar to the situation in $\mathrm{Hg} 5 d$ photoionization. It should be borne in mind that the error bars for the $\beta$ parameter derived from the spin-polarization measurements (closed circles) taken with a bandwidth of $0.3 \mathrm{~nm}$, mainly represent statistical counting errors (single standard deviation). The measurements show that the minimum in $\beta$, which seems to be a sharp structure with a width of about $2-3 \mathrm{eV}$ occurs clearly above the ${ }^{2} D_{3 / 2}$ threshold. This is confirmed also by the calculated values of the spin parameter $\alpha$ (see Sec. III A), though the absolute values are considerably smaller than the RRPA values.

For comparison with the experimental data, theoretical
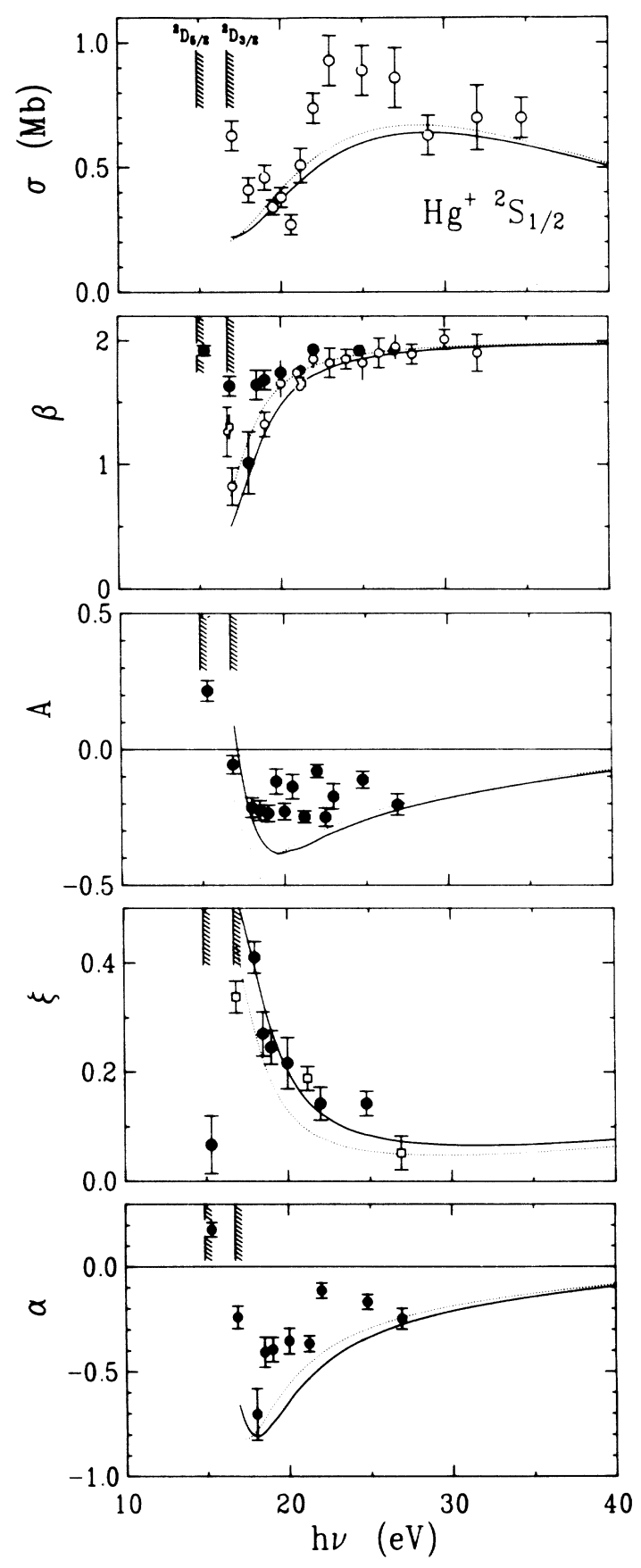

FIG. 1. Photoionization of the $\mathrm{Hg} 6 s$ shell in the region of the Cooper minimum. Cross section $\sigma$ (Ref. 9). $\beta$ parameter: closed circles, this work, open circles, Ref. 13: open squares, Ref. 10: open triangle, Ref. 8. Spin parameters $A, \alpha$, and $\xi$ : closed circles, this work; open squares, Ref. 10. Solid curve, RRPA length form; dotted curve, RRPA velocity form. The dashed lines indicate the ${ }^{2} D_{5 / 2}$ and ${ }^{2} D_{3 / 2}$ thresholds. 
results of the RRPA in length form (solid curve) and velocity form (dotted curve) are given in Fig. 1. We refer to Ref. 10 for a discussion of the earlier RRPA calculation, ${ }^{5}$ the RPAE calculation ${ }^{31}$ (for $\sigma$ only, since nonrelativistic theory gives automatically $\xi=0$ and $\beta=2$ ), and a calculation in the Tamm-Dankoff approximation (TD). ${ }^{5}$ The general energy dependence of all photoionization parameters is very well described by all the calculations, except for the position of the extrema in $\sigma, \beta, A$, and $\xi$, which are experimentally found a few $\mathrm{eV}$ above the ${ }^{2} D_{3 / 2}$ threshold. An RTDLDA calculation ${ }^{6}$ also fails to reproduce the minimum above threshold. Surprisingly, only the TD calculation ${ }^{5}$ gives the correct position (which is probably accidental since the TD does not include all ground-state correlations but includes the same final-state correlations as accounted for by the RRPA).

In this context it might be of interest to note that in the energy region from the ${ }^{2} D_{3 / 2}$ threshold to approximately $24 \mathrm{eV}$ a great number of resonances attributed to two-electron excitations have been observed in absorption measurements. ${ }^{32}$ In addition, satellites appear in the photoelectron spectrum, ${ }^{33,34}$ which are believed to reflect $6 p^{2}$ admixtures in the $6 s^{2}$ ground state with ${ }^{2} P_{1 / 2}$ and ${ }^{2} P_{3 / 2}$ final ionic states. Figure 2 shows a photoelectron spectrum taken at a photon energy of $20.77 \mathrm{eV}$, where the satellite peak ${ }^{2} P_{3 / 2}$ appears with considerable intensity. The $p_{1 / 2}$ electrons, expected with a kinetic energy of 120 meV lower than the $d_{3 / 2}$ electrons, have not been observed, probably due to the relatively broad bandpass chosen for the electron spectrometer. The intensity of the satellite line is very sensitive to small changes in the photon energy. At certain wavelengths the satellite is drastically depressed, when the photon energy is changed by only one bandpass of the monochromator. In addition, the intensities of the other photoionization channels exhibit variation. This is demonstrated in Table $\mathrm{I}$, where the mutual branching ratios for photoionization of $\mathbf{H g}$ with ${ }^{2} D_{5 / 2},{ }^{2} D_{3 / 2},{ }^{2} S_{1 / 2}$, and ${ }^{2} P_{3 / 2}$ final ionic state are given for some important wavelengths. We used a transmission correction of $1 /\left(E_{\text {trans }}-E_{\text {kin }}\right)$ for the photo-

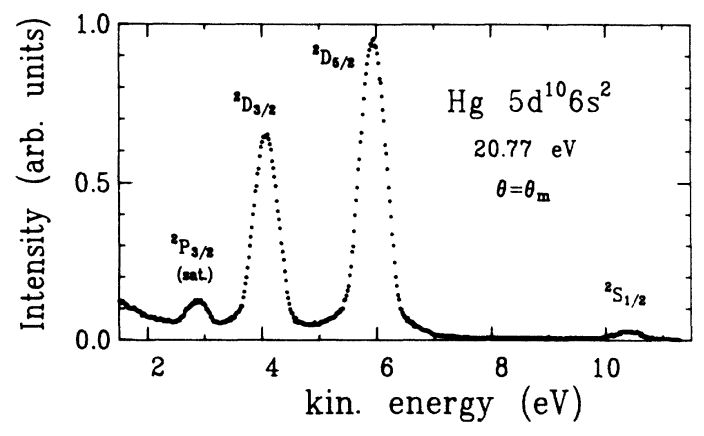

FIG. 2. Photoelectron spectrum of $\mathrm{Hg}\left(5 d^{10} 6 s^{2}\right)$ at the $6 s 6 p 9 d$ double excitation resonance at $h v=20.77 \mathrm{eV}$. The assignments of the final ionic states are given. The ${ }^{2} P_{1 / 2}$ photoelectron line coincides with the ${ }^{2} D_{3 / 2}$ peak to within $0.12 \mathrm{eV}$ (Ref. 32). electron intensities, which has qualitatively, but not thoroughly quantitatively, been checked with xenon as the target gas. We thus cannot give error bars for the absolute values of the branching ratios. The relative variation, however, for small changes of the photon energy by less than $0.5 \mathrm{eV}$ cannot be affected by this approximation.

A more direct demonstration of the rapid change of the cross section with photon energy is given in Fig. 3, where the photoelectron intensities for the ${ }^{2} P_{3 / 2}$ and the ${ }^{2} S_{1 / 2}$ final ionic state in the vicinity of the $6 s$ crosssection minimum are shown. While scanning the photon energy between 19 and $23.5 \mathrm{eV}$, the acceleration potential of the electron spectrometer with fixed transmission energy was simultaneously scanned to detect the satellite line and the $6 s$ photoelectrons, respectively [constant initial state spectroscopy (CISS)]. Figure 3 shows the unnormalized data, i.e., without corrections for monochromator transmission, transmission of the electron optics, and drift of the target pressure. Several structures appear which simultaneously influence the two photoionization channels. Some of the most prominent features can easily be correlated to two-electron excitations as designated by absorption measurements, ${ }^{32}$ especially the members $n=6,7,8,9$ of the $5 d^{9} 6 s 6 p n d$ series. An enlarged graph of the $6 s 6 p 7 d$ resonance, which was denoted as "strong,",32 recorded with photon-energy steps of $10 \mathrm{meV}$ is shown as an inset in Fig. 3. This clearly proves that both the $6 p$ and $6 s$ cross section are considerably modulated at wavelengths where two-electron resonances appear.

Features that so drastically alter the cross section should also influence the other photoionization parameters. We can only speculate that the scattering in the $6 \mathrm{~s}$ cross-section data reported by Ref. 9 or the discrepancies between the different $\beta$ values discussed above might be

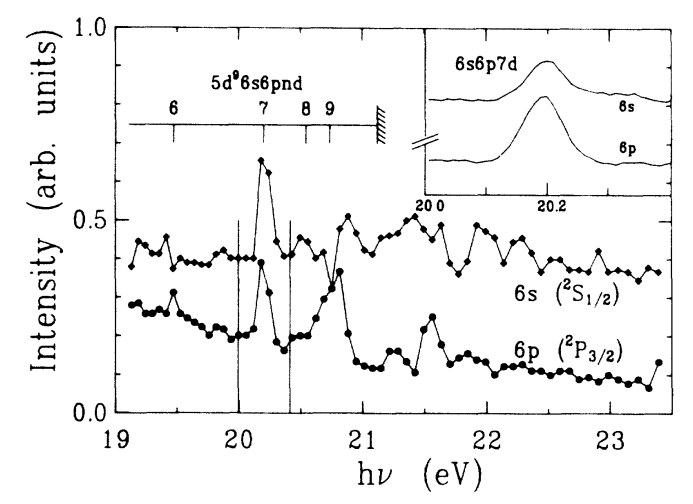

FIG. 3. Photoelectron intensity [constant initial-state spectrum (CISS)] of the $6 s$ photoelectron line and $6 p$ satellite line. The main satellite series $6 s 6 p n d$ is indicated with its limit at $21.13 \mathrm{eV}$. A detailed measurement of the double electron excitation to the $6 s 6 p 7 d$ resonance (the region between the two vertical bars) is shown in the inset. The spectra were recorded at the magic angle $\theta_{\mathrm{mag}}\left(=54.44^{\circ}\right)$ and are not corrected for light intensity, transmission of the monochromator and of the spectrometer, and for drifts of the target pressure. 
TABLE I. Branching ratios for some wavelengths near resonances. The ground-state orbitals are indicated.

\begin{tabular}{cccccc}
\hline \hline$\lambda(\mathrm{nm})$ & $h v(\mathrm{eV})$ & $\frac{I\left(d_{5 / 2}\right)}{I\left(d_{3 / 2}\right)}$ & $\frac{I\left(d_{3 / 2}\right)}{I\left(s_{1 / 2}\right)}$ & $\frac{I\left(d_{3 / 2}\right)}{I\left(p_{3 / 2}\right)}$ & $\frac{I\left(s_{1 / 2}\right)}{I\left(p_{3 / 2}\right)}$ \\
\hline 57.5 & 21.57 & 2.1 & 4.2 & 11.5 & 2.7 \\
58.0 & 21.38 & 2.4 & 3.2 & 40.0 & 12.5 \\
59.7 & 20.77 & 1.9 & 7.1 & 9.1 & 1.3 \\
61.3 & 20.23 & 1.7 & 4.7 & 8.1 & 1.7 \\
61.9 & 20.03 & 1.9 & 7.5 & 67.4 & 9.0 \\
\hline \hline
\end{tabular}

due to such resonances. Some values (e.g., at 22- and 25$\mathrm{eV}$ photon energy) for the spin parameters $A$ and $\xi$ show also unexpected deviations from the general energetic behavior. A closer insight into these phenomena is a subject of further work. In the spin-polarization measurements reported here we consciously tried to avoid wavelengths at which two-electron features are known to appear.

Neither in any theoretical approach, nor in measurements of the cross section and the $\beta$ parameter for $\mathrm{Hg} 6 \mathrm{~s}$ photoionization, have resonances of the kind demonstrated in Fig. 3 been found above the ${ }^{2} D_{3 / 2}$ threshold. Twoelectron excitations are either not accounted for, or their influence upon the $6 s \rightarrow \epsilon p$ channel is underestimated in the models used. Effects that can cause an enhancement of the cross section by a factor of 2 might as well be assumed to be responsible for a theoretically unexpected minimum in the cross section in the same spectral region and may be the reason for the discrepancies between theoretical and experimental results.

\section{MQDT ANALYSIS OF EXPERIMENTAL RESULTS}

According to the dipole selection rules the photoionization of the two outer shells of mercury atoms [ground state $\left.\mathrm{Hg}\left(5 d^{10} 6 s^{2}\right)\left({ }^{1} S_{0}\right)\right]$ reaches eight final states, usually referred to as channels, with $J=1$. They are classified according to the loose coupling or dissociation states and are characterized by the quantum numbers for the ion plus photoelectron system, as given in Table II.

The channels are described by the energy-dependent reduced transition matrix elements $D_{i}$ with the amplitude $D_{i}$ and the phase shift $\delta_{i} \cdot{ }^{35}$ Channels with the same final

TABLE II. Channels arising from dipole excitations of the two outermost, $5 d$ and $6 s$ shells of the ground state $\mathrm{Hg}\left(5 d^{10} 6 s^{2}\right)^{1} S_{0}$.

\begin{tabular}{llc}
\hline \hline $\begin{array}{c}\text { Channel } \\
\text { index } i\end{array}$ & $\begin{array}{c}\text { Transition } \\
\text { (excitation) }\end{array}$ & $\begin{array}{c}\text { Ion }+ \text { electron } \\
\text { state }\end{array}$ \\
\hline 1 & $5 d_{5 / 2} \rightarrow f_{7 / 2}$ & ${ }^{2} D_{5 / 2} f_{7 / 2}$ \\
2 & $5 d_{5 / 2} \rightarrow f_{5 / 2}$ & ${ }^{2} D_{5 / 2} f_{5 / 2}$ \\
3 & $5 d_{5 / 2} \rightarrow p_{3 / 2}$ & ${ }^{2} D_{5 / 2} p_{3 / 2}$ \\
4 & $5 d_{3 / 2} \rightarrow f_{5 / 2}$ & ${ }^{2} D_{3 / 2} f_{5 / 2}$ \\
5 & $5 d_{3 / 2} \rightarrow p_{3 / 2}$ & ${ }^{2} D_{3 / 2} p_{3 / 2}$ \\
6 & $5 d_{3 / 2} \rightarrow p_{1 / 2}$ & ${ }^{2} D_{3 / 2} p_{1 / 2}$ \\
7 & $6 s_{1 / 2} \rightarrow p_{3 / 2}$ & ${ }^{2} S_{1 / 2} p_{3 / 2}$ \\
8 & $6 s_{1 / 2} \rightarrow p_{1 / 2}$ & ${ }^{2} S_{1 / 2} p_{1 / 2}$ \\
\hline
\end{tabular}

ionic state are energy degenerate. Since only differences of phase shifts are physically relevant and a phase-shift difference is not defined between channels with different final ionic state the process of photoionization is described by the 13 independent quantities - five parameters for the $\mathrm{Hg}^{2} \mathrm{D}_{5 / 2}$ final ionic state:

$$
D_{1}, D_{2}, D_{3}, \delta_{1}-\delta_{2}, \delta_{2}-\delta_{3},
$$

five parameters for the $\mathrm{Hg}^{2} D_{3 / 2}$ final ionic state:

$$
D_{4}, D_{5}, D_{6}, \delta_{4}-\delta_{5}, \delta_{5}-\delta_{6},
$$

and three parameters for the $\mathrm{Hg}^{2} S_{1 / 2}$ final ionic state:

$$
D_{7}, D_{8}, \delta_{7}-\delta_{8} \text {. }
$$

These 13 parameters are accessible by the same number of independent observables. It is worthwhile to note that for photoionization of $s$ shells only three independent measurements are necessary for a full characterization, enabling a prediction of other photoionization parameters.

In the following the determination of these parameters is discussed on the basis of the experimental photoionization cross section $\sigma$, the asymmetry parameter $\beta$, and the three spin-polarization parameters $A, \alpha$, and $\xi$. Their dependence from the matrix elements and phase-shift differences was derived from the general relations of Huang $^{36}$ and is listed in the Appendix [Eqs. (A1)-(A15)]. The relation between the spin parameters used in this work and those used by Huang (the Cartesian coordinates, parametrized by $\xi, \eta$, and $\xi$ ) is given in the following equations:

$$
\begin{aligned}
& A=\left(\zeta-2 \xi_{\text {Huang }}\right) / 3, \\
& \xi_{\text {thiswork }}=\eta / 2, \\
& \alpha=-2\left(\zeta+\xi_{\text {Huang }}\right) / 3 .
\end{aligned}
$$

The cross section $\sigma$ is the only parameter that is dependent on the absolute value of the amplitudes, while the others contain only ratios of amplitudes. The $\xi$ parameter is a pure interference term and depends on the sine of the phase-shift differences, while the others contain the cosine. Note that the Fano effect parameter $A$ is dependent only on the phase-shift difference between continua of the same orbital angular momentum.

The "pseudoexperimental" values of the quantities in Eqs. (1) - (3) were determined by a computer code written for this purpose. ${ }^{37}$ For each respective final ionic state 
${ }^{2} D_{5 / 2},{ }^{2} D_{3 / 2}$, and ${ }^{2} S_{1 / 2}$ the code determines numerically the solution vector composed of quantites (1)-(3):

$x=\left(D_{i}, D_{i+1}, D_{i+2}, \delta_{i}-\delta_{i+1}, \delta_{i+1}-\delta_{i+2} \quad(i=1,4)\right.$

for final ionic $D$ states, and

$$
x=\left(D_{7}, D_{8}, \delta_{7}-\delta_{8}\right)
$$

for the final ionic $S$ state, by minimizing the function

$$
\boldsymbol{G}(\boldsymbol{x})=\sum_{k}\left[F_{k}^{\mathrm{expt}}-F_{k}(x)\right]^{2}=\sum_{k} g_{k}
$$

and its derivative up to a certain stopping criteria (method of gradients). The functions $F_{k}(x)(k=1, \ldots, 5)$ are the five dynamical parameters $\sigma, \beta, \xi, A, \alpha$ given for each final ionic state by Eqs. (A1)-(A15) in the Appendix, and $F_{k}^{\text {expt }}$ are the corresponding experimental values.

The phase shift $\delta_{i}$ is related to the quantum defect $\mu_{i}$ by the equation

$$
\delta_{i}=\sigma_{l}+\pi \mu_{i}-l \pi / 2,
$$

$\sigma_{l}$ is the well-known Coulomb phase, the phase shift for an outgoing partial wave with angular momentum $l$ in a pure Coulomb field. $\sigma_{l}$ can be calculated analytically (see, e.g. Ref. 38). In the following only the quantum defects are given by subtracting the Coulomb phase and the term $l \pi / 2$ which satisfies the sign convention for the matrix elements from the phase shift according to Eq. (10). $\mu$ directly indicates the deviation of the true potential from a pure Coulomb potential.

The results of the new RRPA calculation in terms of the eigenchannel parameters $D_{\alpha}$ (close-coupling transition amplitudes) and $\mu_{\alpha}$ (eigenquantum defects) were transformed into the loose coupling parameters $D_{i}$ and $\mu_{i}$ by the use of the energy-dependent transformation matrix $U_{i \alpha}($ Refs. 28 and 35)

$$
D_{i} e^{i \pi \mu_{\iota}}=\sum_{\alpha} U_{i \alpha} D_{\alpha} e^{i \pi \mu_{\alpha}}
$$

In the RRPA calculation experimental thresholds were used.

\section{A. Transition matrix elements for $\mathrm{Hg} 6 s$ photoionization}

From the experimental values of the spin parameters $A$ and $\xi$, the ratio $D_{8} / D_{7}=D$ of the dipole-transition matrix elements and the phase-shift difference $\delta_{7}-\delta_{8}$ can be analytically determined by use of the relations (A13) and (A14) of the Appendix. Since the Coulomb phase difference for the same angular momentum $l$ is zero, the quantum-defect difference is simply determined by

$$
\delta_{i}=\pi \mu_{l} \text {. }
$$

The results deduced from the experimental values are given in the upper two panels of Fig. 4. The general variation of these quantities with photon energy is in agreement with the behavior expected from theoretical considerations. $^{1,4,10}$ The ratio $D_{8} / D_{7}$ is smaller than $1 / \sqrt{2}$ on the low-energy side of the Cooper minimum, then reaches a maximum value and again decreases towards $1 / \sqrt{2}$ with increasing photon energy. [Note that far from the minimum region in the nonrelativistic approximation the ratio of the nonreduced radial matrix elements $R_{8} / R_{7}$ is unity $\left(R_{8}=R_{7}=R_{p}\right){ }^{4}$ This corresponds to a ratio of $1 / \sqrt{2}$ for the reduced matrix elements $D_{i}$.] Similarly, the positive quantum-defect difference $\mu_{7}-\mu_{8}$, which directly reflects the positive sign of the spin parameter $\xi$, goes through a maximum in the Cooperminimum region and decreases to values close to zero far from the extremum. The maximum for both quantities is reached at about $1 \mathrm{eV}$ above the ${ }^{2} D_{3 / 2}$ threshold. The values for the $\beta$ and $\alpha$ parameter presented in Fig. 1 (closed circles) were obtained by putting these results into Eqs. (A12) and (A15), respectively.

The absolute values for $D_{8}$ and $D_{7}$ can only be calcu-

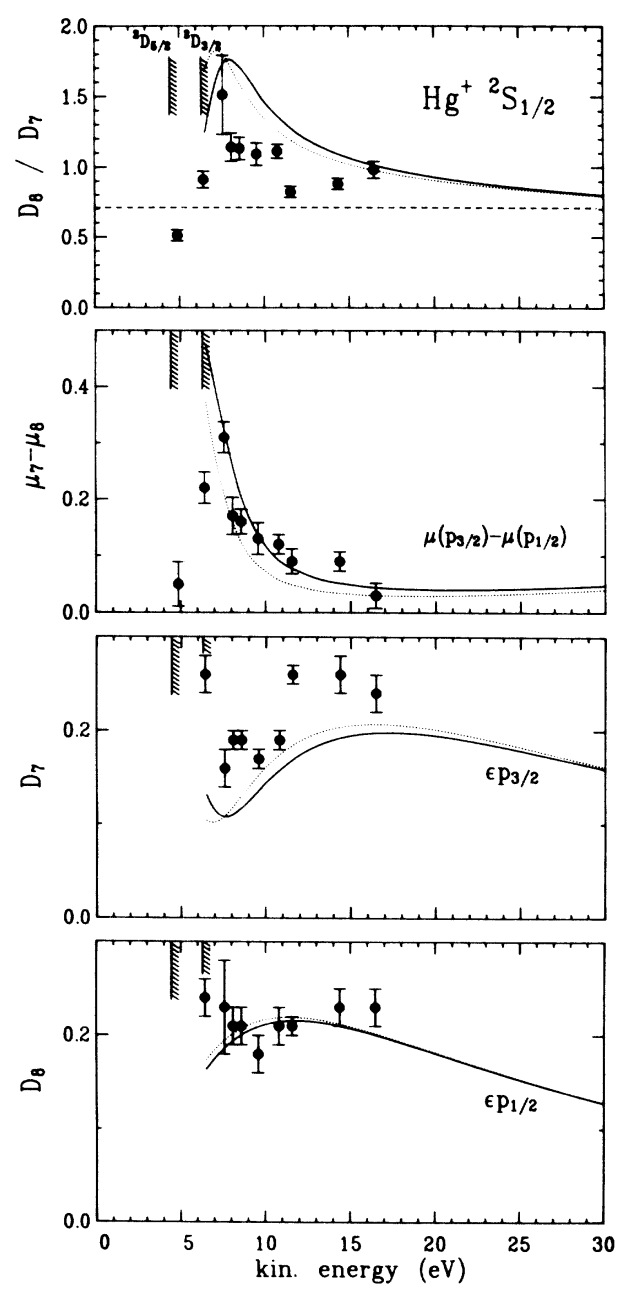

FIG. 4. Ratio of the matrix elements $D_{8} / D_{7}$, the quantumdefect difference and the matrix elements $D_{7}$ and $D_{8}$ for $\mathrm{Hg} 6 \mathrm{~s}$ photoionization. Closed circles, "experimental" values; solid line, RRPA length form; dotted line, RRPA velocity form. The dashed line corresponds to the "nonrelativistic" ratio of $1 / \sqrt{2}$ for $D_{8} / D_{7}$. 
lated by reference to the experimental data of the cross section, 9 according to Eq. (A11). The results are shown in the lower two sections of Fig. 4. $D_{7}$ goes through a minimum in the region between 1 and $4 \mathrm{eV}$ above the ${ }^{2} D_{3 / 2}$ threshold. A minimum at approximately $3 \mathrm{eV}$ above threshold is also apparent in the data for $D_{8}$. It is surprising that the $\epsilon p_{1 / 2}$ amplitude has its minimum at higher photon energies than the $\epsilon p_{3 / 2}$ amplitude, since calculations usually give the opposite order. ${ }^{4,5}$ The results of the RRPA calculation, also given in Fig. 4, are in reasonable agreement for all quantities shown except for the quantum-defect difference $\mu_{7}-\mu_{8}$, where the experimentally established maximum above the ${ }^{2} D_{3 / 2}$ threshold is not correctly reproduced. Consequently, the qualitative behavior of $\xi$ is not matched by the RRPA. The fact that the RRPA fails to produce a minimum in the cross section can be traced to the energy dependence of $D_{8}$, where the RRPA in the critical region gives values which are slightly too large and increase too strongly, thus overcompensating the minimum in $D_{7}$.
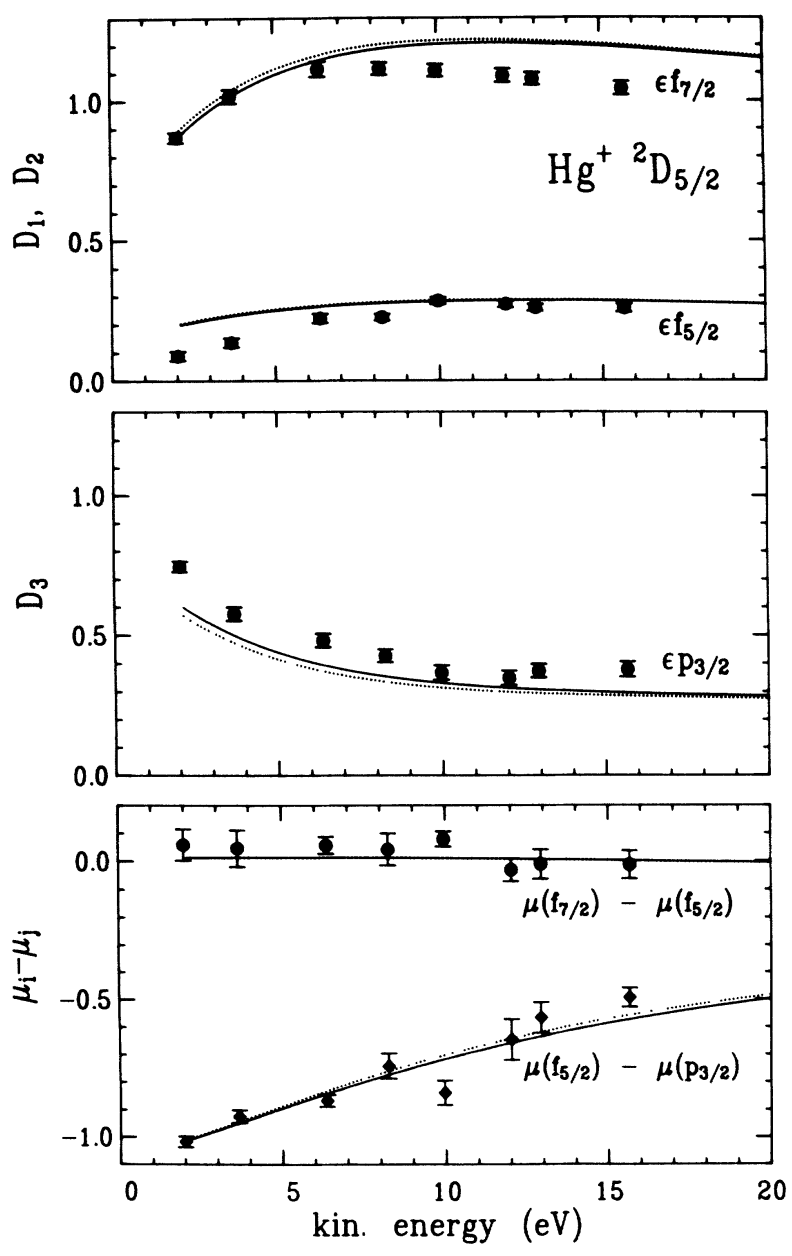

FIG. 5. Matrix elements $D_{1}, D_{2}$, and $D_{3}$ and quantum-defect differences for $\mathrm{Hg} 5 d^{2} \mathrm{D}_{5 / 2}$ final ionic state. Closed circles, "experimental" values; solid line, RRPA length form; dotted line, RRPA velocity form.

\section{B. Transition matrix elements for $\mathrm{Hg} 5 d$ photoionization}

For the evaluation of the $D_{i}$ and $\delta_{i}$ for the $j=5 / 2$ and $j=3 / 2$ levels of the $5 d$ shell the experimental results for the cross section ${ }^{9}$ and for the $\beta$ parameter ${ }^{13}$ utilizing synchrotron radiation were used. The spin-polarization data for $A, \alpha$, and $\xi$ of Ref. 17 and the $\xi$ values of Ref. 16 were taken. In some cases interpolated values for $\sigma$ and $\beta$ were used. The results for the transition matrix elements and quantum-defect differences are displayed in Fig. 5 for the ${ }^{2} D_{5 / 2}$ and in Fig. 6 for the ${ }^{2} D_{3 / 2}$ final ionic state, respectively, as a function of the kinetic energy. The top panel in each figure displays the $5 d \rightarrow \epsilon f$ amplitudes, the middle part the $d \rightarrow \epsilon p$ amplitudes, and the bottom shows the two quantum-defect differences between channels of the same angular momentum $(f-f$ and $p-p)$, and between channels of different $l(p-f)$. The curves represent the new RRPA calculation, both in length (solid) and velocity form (dotted).
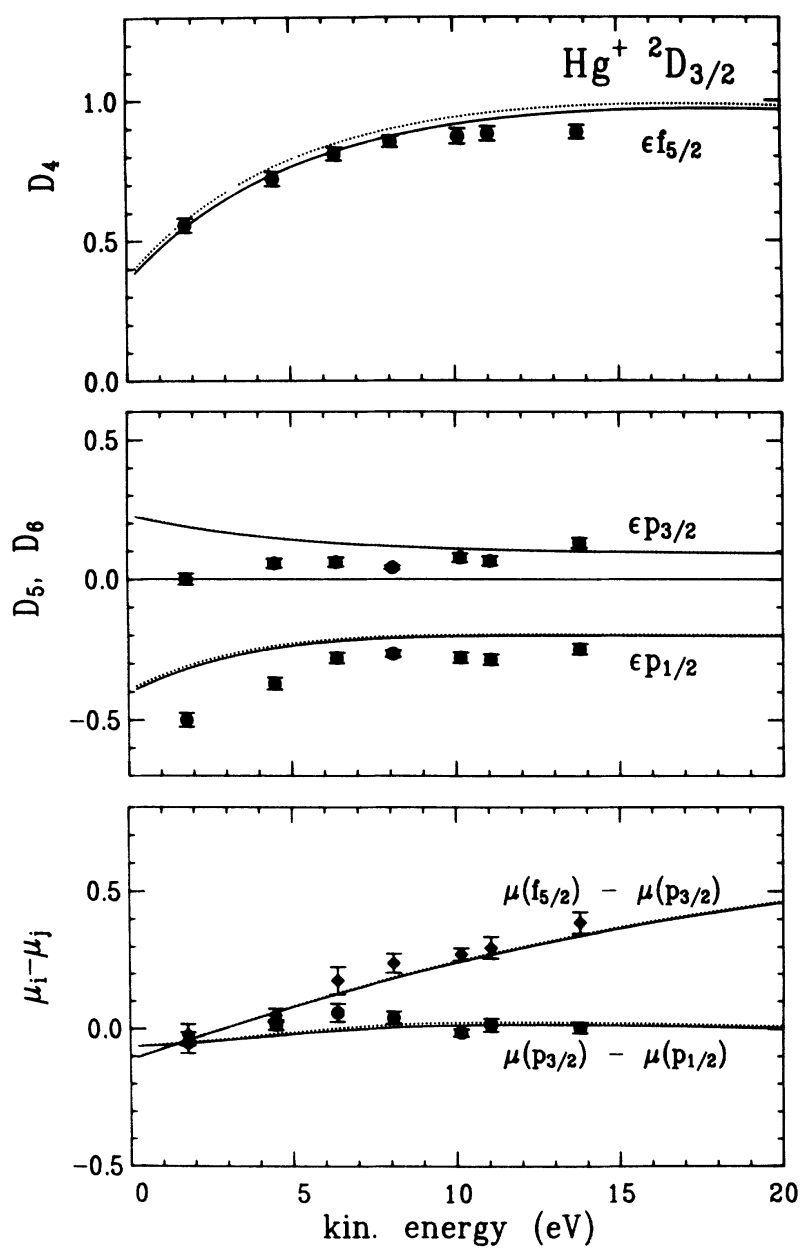

FIG. 6. Matrix elements $D_{4}, D_{5}$, and $D_{6}$ and quantum-defect differences for $\mathrm{Hg} 5 d^{2} D_{3 / 2}$ final ionic state. Closed circles, "experimental" values; solid line, RRPA length form; dotted line, RRPA velocity form. 
All results show a slowly varying dependence on the kinetic energy, the variations being largest close to threshold. The $5 d \rightarrow \epsilon f\left(j=l+\frac{1}{2}\right)$ amplitudes $D_{1}$ and $D_{4}$ are the largest: thus the shape of the cross-section curve is essentially given by the $l \rightarrow l+1$ transitions. The delayed onset due to the potential barrier effect at approximately $10-15 \mathrm{eV}$ above threshold is clearly seen in all $5 d \rightarrow \epsilon f$ channels. The absolute values for the $p$ amplitudes $D_{3}$ and $D_{6}$, however, decrease with increasing kinetic energy. The relativistic quantum-defect differences between the $p$ waves $(j=3 / 2$ and 1/2) (Fig. 6) and the $f$ waves $(j=7 / 2$ and $5 / 2$ ) (Fig. 5) correspond nearly to their nonrelativistic value of zero. This means that the influence of the spin-orbit interaction on the continuum states seems to be restricted to the corresponding matrix elements $D_{1}, D_{2}$ and $D_{6}$. This happens because the excited outer-shell electrons behave almost nonrelativistically at relatively low energies, with small spin-orbit coupling, so that the outer-shell electrons are almost $L S$ coupled. If one considers the transformation matrix $U_{i \alpha}$ for the outer-shell channels, which transforms $j j$-coupled channels to eigenchannels, one can always recognize the approximate $L S$ character of eigenchannels by comparing the matrix with the $L S-j j$ transformation matrix.

The $f-p$ quantum-defect differences increase slowly moving away from threshold. This is in accordance with a recent relativistic Dirac-Slater calculation ${ }^{19}$ where the phase shift $\delta_{f_{7 / 2}}-\delta_{f_{5 / 2}}$ is assumed to be zero and the radial matrix elements to have their nonrelativistic value $R_{7 / 2}=R_{5 / 2}=R_{f}$, since it is argued in Ref. 19 that in the case of the ${ }^{2} D_{5 / 2}$ state the potential barrier effect should prevail strongly over the spin-orbit effect. On the other hand, spin-orbit effects should be more pronounced between the $p$ waves $\left({ }^{2} D_{3 / 2}\right)$, since they are superimposed on a small cross section.

The overall agreement between the "experimental" and the RRPA results is good, although not always within the experimental errors, and the calculated and experimental results show the same trend as a function of energy. The agreement between calculated and experimental values in the present study is considerably better than in a similar study for Ytterbium $4 f^{14} .^{39}$ Some interesting deviations, however, remain. In the case of the ${ }^{2} D_{5 / 2}$ ionic state (Fig. 5) a strong interchannel-transition effect between the $f$ and $p$ waves can be seen which was already observed previously in the $L S$-nonrelativistic picture. ${ }^{23}$ The RRPA underestimates the $p$ amplitude and predicts a monotonically decreasing curve, while the experimental results indicate a minimum around $12-\mathrm{eV}$ kinetic energy. Thus the shape resonance in the $f$ channels is reflected also in the $p$ channel which gains intensity from the $f_{5 / 2}$ channel in the threshold region and from the $f_{7 / 2}$ channel above threshold producing a minimum in between.

For the case of the ${ }^{2} D_{3 / 2}$ ion the situation is similar: the $p$ amplitude $D_{6}(j=1 / 2)$ gets intensity from the $D_{5}(j=3 / 2)$ amplitude which even starts with zero at the threshold. At higher energies RRPA overestimates the $f$ channel again which obviously couples to the $p$ continua. This coupling overcompensates the monotonous decrease in producing a "delayed onset" even in the $p$ continua.
For a discussion of the RPAE calculation ${ }^{31}$ that takes into account this intertransition effect reproducing this minimum and other theories (Hartree-Fock, ${ }^{18}$ HartreeSlater, ${ }^{18}$ and Dirac-Slater calculations ${ }^{19,20}$ ) we refer to Ref. 23.

To visualize the spin-orbit interaction in the continuum more clearly it is useful to consider the nonrelativistic case. In the nonrelativistic model the difference between $\epsilon f_{5 / 2}$ and $\epsilon f_{7 / 2}$ and between $\epsilon p_{3 / 2}$ and $\epsilon p_{1 / 2}$ is neglected, so that only one transition $5 d_{5 / 2} \rightarrow \epsilon f$ and $5 d_{3 / 2} \rightarrow \epsilon p$ occurs which is described by the matrix elements $D_{f}$ and $D_{p}$, respectively. The transition from the $j j$-coupled $D_{j}$ to the $L S$-coupled $D_{l}$ is given by the following equations: $:^{4,35}$

$$
\begin{aligned}
& D_{1}=\sqrt{20 / 21} D_{f}, \\
& D_{2}=\sqrt{1 / 21} D_{f}, \\
& D_{5}=\sqrt{1 / 6} D_{p}, \\
& D_{6}=\sqrt{5 / 6} D_{p} .
\end{aligned}
$$

(Note that for the nonreduced radial matrix elements the equations read $R_{7 / 2}=R_{5 / 2}=R_{f}$, and $R_{3 / 2}=R_{1 / 2}=R_{p}$.)

Figure 7 shows the nonrelativistic matrix elements $D_{f}$
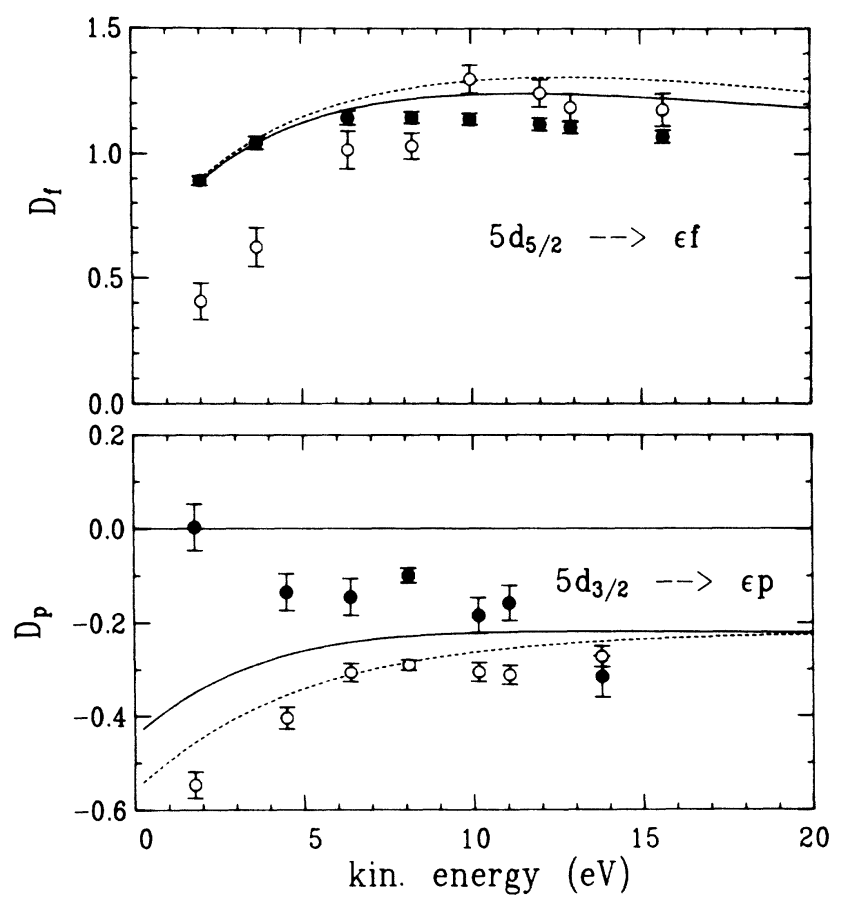

FIG. 7. Nonrelativistic “ $L S$ "-matrix elements $D_{f}$ (top) and $D_{p}$ (bottom) for the $j=5 / 2$ and $j=3 / 2$ subshell, respectively. The data are calculated from the results given in Fig. 5 (top) and in Fig. 6 (middle part) according to Eqs. (13)-(16). Only the length forms of the RRPA curves are given. "Experimental" (closed circles) and RRPA (solid line) results for the $j \rightarrow l+1 / 2$ matrix elements $D_{1}$ and $D_{5}$, and "experimental" (open circles) and RRPA (dashed line) results for the $j \rightarrow l-1 / 2$ matrix elements $D_{2}$ and $D_{6}$. 
(top) and $D_{p}$ (bottom) determined from the relativistic $D_{i}$ of Figs. 5 and 6 according to Eqs. (13)-(16). This figure is thus a quantitative indication of relativistic effects, visible in the difference between the open and closed symbols and between the two curves (only the length form of the RRPA is given). For the $j=5 / 2$ subshell the spin-orbit effect taken into account by the RRPA manifests itself only far from threshold, while the situation for the other subshell is opposite: here only in the threshold region differences appear. The experimental results, however, indicate strong deviations from the nonrelativistic treatment near the threshold, both for the $j=3 / 2$ and the $j=5 / 2$ subshell. At higher kinetic energies spin-orbit effects tend to be less important.

\section{DISCUSSION, CONCLUSIONS, AND SUMMARY}

We have presented an eight-channel MQDT analysis of $\mathrm{Hg} 5 d^{10} 6 s^{2}$ photoionization based on a "complete" experimental data set consisting of photoelectron intensity and spin-polarization data. This analysis has the advantage that different electronic interactions can be seen directly through matrix elements and quantum defects instead of interpreting the interactions through their effect on the measurable quantities such as intensity and spin polarization, which are rather complex functions of these basic quantities. The $5 d$ shell photoionization is dominated by the potential barrier effect in all $d \rightarrow \epsilon f$ partial continua which leads to a shape resonance approximately $10-15 \mathrm{eV}$ above threshold. Spin-orbit interaction in the continuum states leads to an unusual distribution of oscillator strength between the $j=3 / 2$ and $j=1 / 2 p$ partial waves and to a breakdown of the nonrelativistic approximation within the threshold region. The corresponding quantum-defect differences, however, tend to their nonrelativistic value of zero. Interchannel coupling between the $f$ and $p$ partial waves results in some quantiative mismatch of the "measured" intensities to the RRPA prediction.

Measured values of the dynamical parameters for $\mathbf{H g}$ $6 s$ photoionization in the vicinity of the Cooper minimum show good agreement with theoretical data, although for $\sigma, \beta$, and $\xi$ the experimentally found extrema above the ${ }^{2} D_{3 / 2}$ threshold are not correctly reproduced by RPAE, RRPA, and RTDLDA models. This mismatch is reflected in the behavior of the matrix elements and the quantum-defect difference for the $\epsilon p_{1 / 2}$, and $\epsilon p_{3 / 2^{-}}$ continuum states. The evaluation of the experimental data results in a maximum in the quantum-defect difference and a minimum for both transition matrix elements above the ${ }^{2} D_{3 / 2}$ threshold, whereas a refined RRPA calculation only reproduces the minimum above threshold for the $\epsilon p_{3 / 2}$ amplitude. We observed strong modulation of the cross section for direct $6 \mathrm{~s}$ photoionization by about a factor of 2 in the vicinity of two-electron excitations, which are known to occur in the regarded photon-energy range. In addition, the minima in the transition amplitudes occur in unorthodox order (in the $\epsilon p_{1 / 2}$ channel at higher photon energy than in the $\epsilon p_{3 / 2}$ channel). We thus point out that the photoionization cross section of mercury above the $5 d$ thresholds is not a smooth function of photon energy, which in the past was assumed in theoretical considerations and interpretation of experimental results. It should be pointed out that this statement is valid not only for the $6 s$ photoionization, but also - to a lesser extent due to the larger cross sectionfor the $5 d$ photoionization and thus may also be responsible for the difficulties in reproducing the experimental data in the threshold region. For $6 s$ photoionization this leads to the conclusion that the minimum in the cross section at about $20-\mathrm{eV}$ photon energy reported in Ref. 9 and usually denoted as a Cooper minimum is caused by combination of the energy dependence of the direct $6 s \rightarrow \epsilon p$ transition matrix elements and the influence of resonances due to two-electron excitations. Further investigations in this spectral region should yield a deeper insight into the complex processes accompanied by twoelectron excitations. From an experimentalist's point of view, a relative determination of the cross section or measurements of the $\beta$ parameter are best suited for this purpose; spin-polarization experiments suffer from the intensity loss in Mott scattering and are particularly difficult in $\mathrm{Hg} 6 s$ photoionization with a cross section of less than $0.5 \mathrm{Mb}$, resulting in accumulation times of $20 \mathrm{~h}$ and more for the measurement of a single spin-polarization value. We also hope that the phenomena observed in this system should be a challenge to develop more extensive theoretical models, since in this case the influence of two-electron excitations even on a basic quantity like the partial cross section has obviously been underestimated.

\section{ACKNOWLEDGMENTS}

Financial support of the Bundesministerium für Forschung und Technologie is gratefully acknowledged (Grant Nos. 5331 and 5431 AX). The work of V. R. was partially supported by the National Science Foundation. V. R. would also like to acknowledge a computational grant from the Academic Computing Center of the University of Virginia.

\section{APPENDIX}

The dynamical photoionization parameters are $\sigma$ (cross section), $\beta$ (angular distribution asymmetry) and the three spin-polarization parameters $A, \alpha$, and $\xi$ for $d_{5 / 2}, d_{3 / 2}$, and $s$ shells as a function of the reduced matrix elements $D_{1}$ and the phase-shift differences $\delta_{i}-\delta_{j}$. $\alpha, a_{0}$, and $\omega$ denote the fine-structure constant, the Bohr radius, and the photon energy in atomic units, respectively (1 a.u. = 2 Ry). We have

$$
\begin{aligned}
& \sigma_{5 / 2}=4 \pi^{2} \alpha a_{0}^{2} \omega\left(D_{1}^{2}+D_{2}^{2}+D_{3}^{2}\right), \\
& \beta_{5 / 2}=\frac{-25 D_{1}^{2}-32 D_{2}^{2}+7 D_{3}^{2}+12 \sqrt{5} D_{1} D_{2} \cos \left(\delta_{1}-\delta_{2}\right)-12 \sqrt{70} D_{1} D_{3} \cos \left(\delta_{1}-\delta_{3}\right)-6 \sqrt{14} D_{2} D_{3} \cos \left(\delta_{2}-\delta_{3}\right)}{35\left(D_{1}^{2}+D_{2}^{2}+D_{3}^{2}\right)},
\end{aligned}
$$




$$
\begin{aligned}
& \xi_{5 / 2}=\frac{-42 \sqrt{5} D_{1} D_{2} \sin \left(\delta_{1}-\delta_{2}\right)+12 \sqrt{70} D_{1} D_{3} \sin \left(\delta_{1}-\delta_{3}\right)-15 \sqrt{14} D_{2} D_{3} \sin \left(\delta_{2}-\delta_{3}\right)}{140\left(D_{1}^{2}+D_{2}^{2}+D_{3}^{2}\right)}, \\
& A_{5 / 2}=\frac{9 D_{1}^{2}-2 D_{2}^{2}-7 D_{3}^{2}-8 \sqrt{5} D_{1} D_{2} \cos \left(\delta_{1}-\delta_{2}\right)}{14\left(D_{1}^{2}+D_{2}^{2}+D_{3}^{2}\right)} \\
& \alpha_{5 / 2}=\frac{15 D_{1}^{2}-8 D_{2}^{2}-7 D_{3}^{2}+10 \sqrt{5} D_{1} D_{2} \cos \left(\delta_{1}-\delta_{2}\right)+21 \sqrt{14} D_{2} D_{3} \cos \left(\delta_{2}-\delta_{3}\right)}{35\left(D_{1}^{2}+D_{2}^{2}+D_{3}^{2}\right)} \text {, } \\
& \sigma_{3 / 2}=4 \pi^{2} \alpha a_{0}^{2} \omega\left(D_{4}^{2}+D_{5}^{2}+D_{6}^{2}\right) \text {, } \\
& \beta_{3 / 2}=\frac{4 D_{4}^{2}-4 D_{5}^{2}+6 D_{4} D_{5} \cos \left(\delta_{4}-\delta_{5}\right)-6 \sqrt{5} D_{4} D_{6} \cos \left(\delta_{4}-\delta_{6}\right)-2 \sqrt{5} D_{5} D_{6} \cos \left(\delta_{5}-\delta_{6}\right)}{5\left(D_{4}^{2}+D_{5}^{2}+D_{6}^{2}\right)} \text {, } \\
& \xi_{3 / 2}=\frac{15 D_{4} D_{5} \sin \left(\delta_{4}-\delta_{5}\right)-6 \sqrt{5} D_{4} D_{6} \sin \left(\delta_{4}-\delta_{6}\right)+3 \sqrt{5} D_{5} D_{6} \sin \left(\delta_{5}-\delta_{6}\right)}{20\left(D_{4}^{2}+D_{5}^{2}+D_{6}^{2}\right)} \text {, } \\
& A_{3 / 2}=\frac{-3 D_{4}^{2}+2 D_{5}^{2}+D_{6}^{2}-4 \sqrt{5} D_{5} D_{6} \cos \left(\delta_{5}-\delta_{6}\right)}{6\left(D_{4}^{2}+D_{5}^{2}+D_{6}^{2}\right)}, \\
& \alpha_{3 / 2}=\frac{-12 D_{4}^{2}+2 D_{5}^{2}+10 D_{6}^{2}+27 D_{4} D_{5} \cos \left(\delta_{4}-\delta_{5}\right)+5 \sqrt{5} D_{5} D_{6} \cos \left(\delta_{5}-\delta_{6}\right)}{15\left(D_{4}^{2}+D_{5}^{2}+D_{6}^{2}\right)} \text {, } \\
& \sigma_{s}=4 \pi^{2} \alpha a_{0}^{2} \omega\left(D_{7}^{2}+D_{8}^{2}\right) \text {, } \\
& \beta_{s}=\frac{D_{7}^{2}+2 \sqrt{2} D_{7} D_{8} \cos \left(\delta_{7}-\delta_{8}\right)}{D_{7}^{2}+D_{8}^{2}} \text {, } \\
& \xi_{s}=\frac{3 D_{7} D_{8} \sin \left(\delta_{7}-\delta_{8}\right)}{2 \sqrt{2}\left(D_{7}^{2}+D_{8}^{2}\right)} \\
& A_{s}=\frac{5 / 2 D_{7}^{2}-D_{8}^{2}-2 \sqrt{2} D_{7} D_{8} \cos \left(\delta_{7}-\delta_{8}\right)}{3\left(D_{7}^{2}+D_{8}^{2}\right)}, \\
& \alpha_{s}=\frac{D_{7}^{2}-4 D_{8}^{2}+\sqrt{2} D_{7} D_{8} \cos \left(\delta_{7}-\delta_{8}\right)}{3\left(D_{7}^{2}+D_{8}^{2}\right)} \text {. }
\end{aligned}
$$

${ }^{1}$ N. A. Cherepkov, Phys. Lett. 66A, 204 (1978).

${ }^{2}$ W. R. Johnson and K. T. Cheng, Phys. Rev. A 20, 978 (1979); K. N. Huang, W. R. Johnson, and K. T. Cheng, Phys. Rev. Lett. 43, 1658 (1979); At. Data Nucl. Data Tables 26, 33 (1981).

${ }^{3}$ K. T. Cheng, K. -N. Huang, and W. R. Johnson, J. Phys. B 13, L45 (1980).

${ }^{4}$ N. A. Cherepkov, Adv. At. Mol. Phys. 19, 395 (1983).

${ }^{5}$ W. R. Johnson, V. Radojević, P. Deshmuk, and K. T. Cheng, Phys. Rev. A 25, 337 (1982).

${ }^{6}$ F. A. Parpia and W. R. Johnson, J. Phys. B 16, L375 (1983); 17, 531 (1984).

${ }^{7}$ F. A. Parpia, W. R. Johnson, and V. Radojević, Phys. Rev. A 29, 3173 (1984).

${ }^{8}$ A. Niehaus and M. W. Ruf, 252, 84 (1972).

${ }^{9}$ S. P. Shannon and K. Codling, J. Phys. B 11, 1193 (1978).

${ }^{10} \mathrm{G}$. Schönhense, U. Heinzmann, J. Kessler, and N. A. Cherepkov, Phys. Rev. Lett, 48, 603 (1982).

${ }^{11}$ A. Fahlmann, T. A. Carlson, and M. O. Krause, Phys. Rev. Lett. 50, 1114 (1983).

${ }^{12}$ H. Derenbach and V. Schmidt, J. Phys. B 16, L337 (1983).

${ }^{13}$ B. H. McQaide, M. S. Banna, P. Gerard, and M. O. Krause, Phys. Rev. A 35, 1603 (1987).
${ }^{14}$ M. Müller, F. Schäfers, N. Böwering, Ch. Heckenkamp, U. Heinzmann, BESSY Annual Report, 1985 (unpublished), p. 79.

${ }^{15}$ G. Schönhense, J. Phys. B 14, L187 (1981).

${ }^{16} \mathrm{G}$. Schönhense, F. Schäfers, U. Heinzmann, and J. Kessler, Z. Phys. A 304, 31 (1982).

${ }^{17}$ F. Schäfers, Ch. Heckenkamp, G. Schönhense, and U. Heinzmann, J. Phys. B 21, 769 (1988).

${ }^{18}$ F. Keller and F. Combet Farnoux, J. Phys. B 12, 2821 (1979); 15, 2657 (1982); Y. A. Kim, R. H. Pratt, A. Ron, and H. K. Tseng, Phys. Rev. A 22, 567 (1980).

${ }^{19}$ F. Keller and F. Combet Farnoux, J. Phys. B 18, 3581 (1985).

${ }^{20}$ E. H. Walker, J. Berkowitz, J. L. Dehmer, and J. I. Waber, Phys. Rev. Lett. 31, 678 (1973).

${ }^{21}$ B. R. Tambe, W. Ong, and S. T. Manson, Phys. Rev. A 23, 799 (1981); C. E. Theodosiou, A. F. Starace, B. R. Tambe, and S. T. Manson, ibid. 24, 301 (1981).

${ }^{22}$ W. R. Johnson and V. Radojević, Phys. Lett. 92A, 75 (1982).

${ }^{23} \mathrm{G}$. Schönhense and U. Heinzmann, Phys. Rev. A 29, 987 (1984).

${ }^{24}$ W. R. Johnson and C. D. Lin, Phys. Rev. A 20, 964 (1979); W. R. Johnson, C. D. Lin, K. T. Cheng, and C. M. Lee, Phys. Scr. 21, 409 (1980) 
${ }^{25}$ M. Y. Amusia and N. A. Cherepkov, Case Stud, At. Phys. 5, 47 (1975).

${ }^{26}$ H. P. Kelly, Adv. Theor. Phys. 2, 75 (1968); S. L. Karter and H. P. Kelly, J. Phys. B 11, 2476 (1978).

${ }^{27}$ F. Schäfers, W. Peatman, A. Eyers, Ch. Heckenkamp, G. Schönhense, and U. Heinzmann, Rev. Sci. Instrum. 57, 1032 (1986).

${ }^{28} \mathrm{Ch}$. Heckenkamp, F. Schäfers, G. Schönhense, and U. Heinzmann, Z. Phys. D 2, 257 (1986).

${ }^{29} \mathrm{Ch}$. Heckenkamp, A. Eyers, F. Schäfers, G. Schönhense, and U. Heinzmann, Nucl. Instrum. Methods A 246, 500 (1986).

${ }^{30}$ M. Müller, F. Schäfers, N. Böwering, Ch. Heckenkamp, U. Heinzmann, Phys. Scr. 35, 459 (1987).

${ }^{31}$ V. K. Ivanov, S. Yu. Medvedev, and V. A. Sosnivker, Opt. Spektrosk. 58, 749 (1985).

${ }^{32}$ M. W. D. Mansfield, Astrophys. J. 180, 1011 (1973).

${ }^{33}$ S. Süzer, S. T. Lee, and D. A. Shirley, Phys. Rev. A 13, 1842
(1976).

${ }^{34}$ J. Berkowitz, J. L. Dehmer, Y. K. Kim, and J. P. Desclaux, J. Chem Phys. 61, 2556 (1974).

${ }^{35}$ U. Heinzmann, J. Phys. B 13, 4353 (1980); 13, 4367 (1980).

${ }^{36}$ K. N. Huang, Phys. Rev. A 22, 223 (1980).

${ }^{37}$ M. Müller, Ph.D. thesis, Universität Bielefeld, West Germany, 1989.

${ }^{38}$ Handbook of Mathematical Functions, Nat. Bur. Stand. Appl. Math. No. 35, edited by M. Abramowitz and I. A. Stegun (U.S. GPO, Washington, D. C., 1968).

${ }^{39}$ M. Müller, N. Böwering, U. Heinzmann, and V. Radojević, in Proceedings of the 16th International Conference on the Physics of Electronic and Atomic Collisions, New York, 1989, AIP Conf. Proc. No. 205, edited by A. Dalgarno, R. S. Freund, M. S. Lubell, and T. B. Lucatorto (AIP, New York, in press), p. 10. 